\title{
Dynamic wake modeling and state estimation for improved model-based receding horizon control of wind farms
}

\author{
Carl R. Shapiro, ${ }^{1}$ Johan Meyers, ${ }^{2}$ Charles Meneveau, ${ }^{1}$ and Dennice F. Gayme ${ }^{1}$
}

\begin{abstract}
Power tracking is an emerging application for wind farm control designs that allows farms to participate in a wider range of grid services, such as secondary frequency regulation. Control designs that enable large wind farms to follow a time-varying power trajectory are complicated by aerodynamic interactions that make it impossible to decouple upstream wind turbine control actions from downstream power production. This coupling is particularly important in applications where the reference trajectory is changing faster than, or at a similar rate as, the propagation of turbine wakes through the farm. In this work we overcome these difficulties by using a dynamic wake model that accounts for wake expansion, advection, and multi-wake interactions within a model-based receding horizon controller for coordinated control of a large multiturbine wind farm. An ensemble Kalman filter is employed for state estimation and error correction at the turbine level. We implement the controller in high-fidelity numerical simulations of turbulent flow in a wind farm with 84 turbines and then test the controlled farm's ability to track a power reference signal. The results demonstrate the ability of the control algorithm to track two types of power reference signal used by a US independent system operator.
\end{abstract}

\section{INTRODUCTION}

The recent worldwide growth of installed wind capacity has significant implications for wind plant design and control. When operating as niche energy sources, regulators often treat wind energy as a "must take" resource and wind plants prioritize maximizing power production. With growing grid penetration, however, regulators are starting to require wind plants to participate in grid services that maintain grid stability [1], [2]. As a result, wind plants of the future may be required to provide vital grid services for power balancing, such as secondary frequency regulation, in which participating generators track power reference signals sent by the grid operator.

Wind turbine power production can be changed through a variety of aerodynamic actions, such as thrust modulation via blade pitch angle and tip speed ratio [3], yawing [4], or tilting [5]. Coordinated aerodynamic control of wind farms to track a power signal, however, is complicated by

*CRS, CM, and DFG are supported by the National Science Foundation (grant nos: ECCS-1230788 and OISE-1243482, the WINDINSPIRE project). JM is supported by the European Research Council (ActiveWindFarms, grant no: 306471). This research project was conducted using computational resources at the Maryland Advanced Research Computing Center (MARCC).

${ }^{1}$ Carl R. Shapiro, Charles Meneveau, and Dennice F. Gayme are with the Department of Mechanical Engineering, Johns Hopkins University, Baltimore, Maryland 21218, USA crshapiroejhu.edu, meneveauljhu.edu, denniceejhu.edu

${ }^{2}$ Johan Meyers is with the Department of Mechanical Engineering, KU Leuven 3001 Leuven, Belgium johan. meyers@kuleuven.be wind turbine wakes, which are generated as wind turbines extract kinetic energy from the incoming wind. These wakes move downstream and therefore interact with downstream turbines. All the while, turbulence causes the wakes to meander, diffuse, and decrease in strength [6]. Through these phenomena, aerodynamic changes at upstream turbineswhich can alter the location, intensity, and recovery rate of wakes-are coupled with downstream power production. Since this coupling is produced by movement of wakes downstream, control actions at an upstream row of turbines only affect downstream rows at a later time based on the wake advection speed.

Following a power reference signal that changes at speeds commensurate with the travel time of wakes through the farm therefore requires an understanding of important aerodynamic effects. A number of approaches to modeling wake interactions, ranging from high fidelity simulations of the underlying flow physics [6], [7], [8] to engineering models [9], [10], [11], have been considered. While the former is not suitable for real-time control because of computational complexity [12], past engineering models do not capture all of the dynamic phenomena needed for power tracking controllers. Many wake models-such as static wake models [9], [13], [14], wind turbine atmospheric boundary layer models [7], [10], and the coupled wake boundary layer model [11] were developed to capture wake recovery in a time-averaged sense. While these models are useful for designing wind farms, they do not include dynamic wake interactions. As a result, controllers based on these models may fail to provide acceptable power tracking performance [15]. Only recently have dynamic approaches-such as the dynamic wake meandering model [16], reduced-order linear models [17], and dynamic extensions of static wake models [3], [18] — been considered for power tracking control.

In this work we discuss a unified control framework for power tracking with wind farms through thrust modulation. This approach relies on a new dynamic wake model [19], which represents each turbine wake as a one-dimension partial differential equation (PDE) and includes the dynamic effect of thrust modulation through wake advection. An ensemble Kalman filter (EnKF) is used to provide state and parameter estimation for the dynamic wake model. Finally, a model-based receding horizon controller allows the wind farm to track a time-varying power trajectory [19]. Moreover, high-fidelity large eddy simulations (LES) are used as computational "model wind farms" to test the controller and state estimation.

Previous results demonstrated the good tracking perfor- 
mance in many tests of this approach without state and parameter estimation [19], [20]. By incorporating state and parameter estimation, however, this unified approach allows for online estimation of wake model parameters that were fit from pre-control measurements in prior work. This improves the robustness of the control method by allowing the controller to be run continuously without recalculating wake parameters. Another notable improvement over prior work is the inclusion of explicit bounds on the control variables and the introduction of an auxiliary control variable to impose limits of the rate of change of the control.

The remainder of this paper is organized as follows. Section II discusses the problem setup and notation. The controller, state estimation, and underlying dynamic wake model are discussed in Section III. Numerical results are presented and discussed in Section IV. Conclusions are listed in Section V

\section{PROBLEM SETUP}

In the current work, we consider regularly arranged wind farms composed of $N$ rows of $M$ turbines aligned with the prevailing wind direction, as shown in Figure 1. The coordinate aligned with the wind (streamwise) direction is denoted as $x$. The other horizontal (spanwise) direction is denoted as $y$. The streamwise and spanwise extents of the farm are denoted by $L_{x}$ and $L_{y}$, respectively. Each turbine row is located at $x=s_{n}$, and each turbine column is located at $y=y_{m}$.

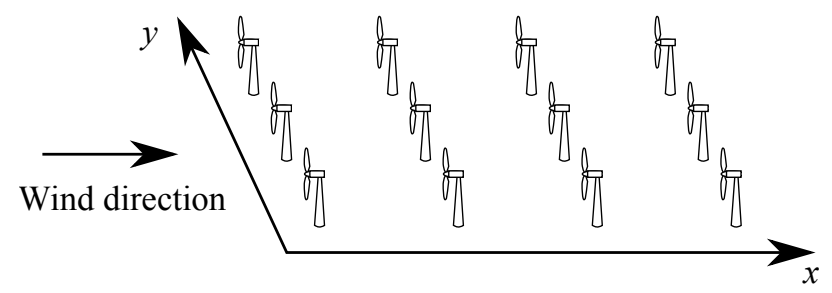

Fig. 1. Diagram of an aligned with farm with $N=4$ rows and $M=3$ columns.

Italicized letters and symbols denote scalars and scalarvalued functions, while boldface indicates matrices, vectors, and vector-valued functions. We denote elements of a vector a as scalars $a_{n}$ and the scalar-valued elements of a vectorvalued function $\mathbf{f}(x)$ as $f_{n}(x)$. Each continuous function includes its argument. An ensemble of vectors is indexed using superscripts placed within parentheses. For example, the vector $\mathbf{v}^{(i)}$ is the vector $\mathbf{v}$ belonging to the $i$-th ensemble member.

\section{MODELING AND CONTROLLER DESIGN}

We propose a closed-loop model-based receding horizon wind farm controller with state estimation. Its purpose is to enable a wind farm to track a power signal $P_{\text {ref }}(t)$. A block diagram of the controlled wind farm system is shown in Figure 2. Each of the control blocks along with their input and output parameters are described in the following sections.

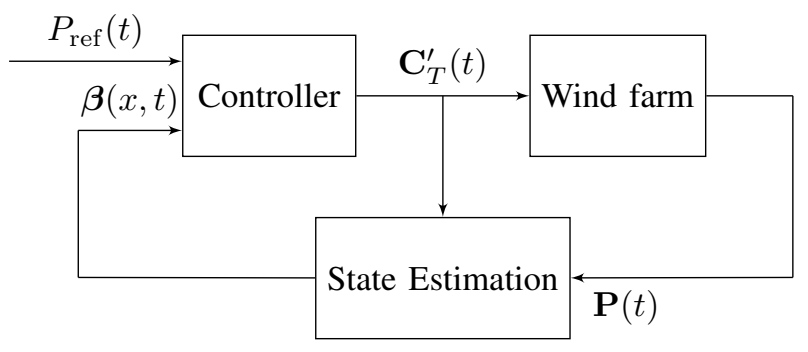

Fig. 2. Controlled wind farm system block diagram showing the modelbased receding horizon controller, state estimation, and wind farm. $P_{\text {ref }}(t)$ is the power reference signal, $\mathbf{C}_{T}^{\prime}(t)$ are the turbine-local thrust coefficients [8], $\mathbf{P}(t)$ is the vector of measured power signals, and $\boldsymbol{\beta}(x, t)$ are model parameters and states.

\section{A. Wake Model}

We now describe the time-varying wake model, first proposed in [19], which is used in both the control and state estimation blocks of Figure 2. This model draws from several elements of the Jensen model approach [9], which assumes linear wake expansion and square-superposition of wake deficits to estimate the velocity field in the farm. Each row of turbines is considered collectively such that the only important spatial coordinate $x$ is in the streamwise direction. First, a wake velocity deficit $\delta u_{n}(x, t)$ for the $n$-th row is computed as if it were subjected to the same freestream velocity $U_{\infty}$ as all other rows. Taking the Lagrangian derivative of the wake as it travels downstream results in the following one-dimensional PDE governing the wake velocity deficit $\delta u_{n}(x, t)$

$$
\frac{\partial \delta u_{n}}{\partial t}+U_{\infty} \frac{\partial \delta u_{n}}{\partial x}=-w_{n}(x) \delta u_{n}(x, t)+f_{n}(x, t) .
$$

The wake decay function

$$
w_{n}(x)=2 \frac{U_{\infty}}{d_{n}(x)} \frac{d}{d x} d_{n}(x)
$$

is specified through the normalized wake diameter function (the wake diameter of the $n$-th row normalized by the rotor diameter $D$ ) that exhibits a linear growth rate of $k_{n}$ behind the turbine

$$
d_{n}(x)=1+k_{n} \ln \left[1+\exp \left(\frac{x-s_{n}-D}{D / 2}\right)\right] .
$$

The forcing function

$$
f_{n}(x, t)=\frac{2 U_{\infty}^{2}}{d_{n}^{2}(x)} \frac{C_{T n}^{\prime}(t)}{4+C_{T n}^{\prime}(t)} G\left(x-s_{n}\right)
$$

captures the effect of the wind turbines on the flow field; i.e. it represents the initial velocity deficit imparted by the turbines onto the flow, or, equivalently, the energy extraction of the turbine. Here the turbine-local thrust coefficient is assumed to parameterize blade pitch angle and tip speed ratio, and

$$
G\left(x-s_{n}\right)=\frac{1}{\Delta \sqrt{2 \pi}} e^{-\frac{\left(x-s_{n}\right)^{2}}{2 \Delta^{2}}}
$$

is a normalized Gaussian smoothing function with $\Delta=D / 2$. 
Again following the Jensen model approach, the squared deficits [9] are superposed to calculate the velocity at position $x$ and time $t$

$$
u(x, t)=U_{\infty}-\left(\sum_{m=1}^{N} \delta u_{m}^{2}(x, t)\right)^{1 / 2} .
$$

The estimated velocity at the $n$-th turbine row $\hat{u}_{n}$ is then found using

$$
\hat{u}_{n}(t)=\int_{0}^{L_{x}} u(x, t) G\left(x-s_{n}\right) d x
$$

where the Gaussian smoothing function is used as the integration kernel. This integral transform is needed to guarantee a smooth forcing term in the adjoint equations used in Section III-C [19]. The velocity at the turbine is then used to calculate the estimated power $\hat{P}_{n}$ of the $M$ turbines in the $n$-th row

$$
\hat{P}_{n}(t)=M \frac{1}{2} \rho \frac{\pi D^{2}}{4} C_{T n}^{\prime}(t) \hat{u}_{n}^{3}(t),
$$

where $\rho$ is the air density.

The inputs of the wake model are the turbine-local thrust coefficient $C_{T n}^{\prime}(t)$. The outputs are the vector of estimated row power production $\hat{P}_{n}(t)$. Acknowledging that the wake expansion rates and freestream velocity may change in time, we now allow these parameters to be time-dependent, and denote the vector of wake model states and parameters as $\beta_{n}(x, t)=\left[\delta u_{n}(x, t), k_{n}(t), U_{\infty}(t)\right]$.

\section{B. State estimation}

The wake model discussed above includes important aspects of wake advection, expansion, and interaction that have significant effects on the total wind farm power production. However, the model makes a number of simplifying assumptions and neglects natural variations in power production due to turbulence within the wind farm. There is also uncertainty in the model parameters, specifically the freestream velocity $U_{\infty}$ and the wake expansion coefficients $k_{n}$ in (3).

In this section we discuss the use of power measurements at the turbine rows $P_{n}(t)$ for error correction and estimation of the wake model states and parameters $\beta_{n}(x, t)=$ $\left[\delta u_{n}(x, t), k_{n}(t), U_{\infty}(t)\right]$, all of which are now allowed to vary in time. The freestream velocity $U_{\infty}(t)$ is estimated using a low-pass filter on the power at the first row, while the wake expansion parameters $k_{n}(t)$ and wake velocity deficits $\delta u_{n}(x, t)$ are estimated using an ensemble Kalman filter [21]. The resulting state estimation block diagram is shown in Figure 3.

This approach makes assumptions about the time scales associated with the wake model states and parameters. Since the freestream velocity $U_{\infty}(t)$ uniformly affects all turbines within the farm, we assume that it represents mesoscale phenomena that change over relatively long time scales compared to the advective scale of the wind farm. In other words, the incoming wind speed changes more slowly than the travel time of the wind through the farm. The other parameters and states, however, do not have the same uniform effect and are

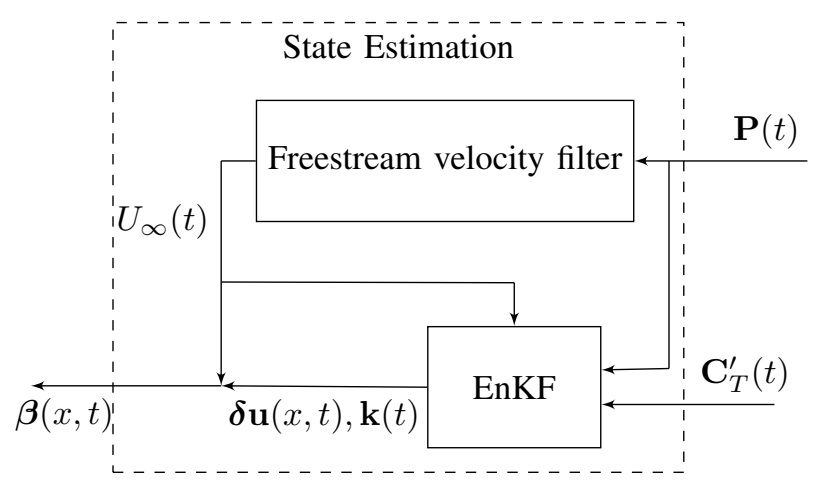

Fig. 3. State estimation block diagram showing ensemble Kalman filter and freestream velocity filter.

allowed to vary over shorter time scales. As a result, the slowly-varying freestream velocity is estimated using a firstorder relaxation of measurements of the power at the first turbine $P_{1}(t)$ with a time constant $\gamma$

$\frac{d U_{\infty}}{d t}=\frac{1}{\gamma}\left(\frac{4+C_{T 1}^{\prime}(t)}{4}\left(\frac{8 P_{1}(t)}{M \rho \pi D^{2} C_{T 1}^{\prime}(t)}\right)^{1 / 3}-U_{\infty}(t)\right)$

Since the wake expansion parameters $k_{n}(t)$ and velocity deficits $\delta u_{n}(x, t)$ vary over shorter time scales, they are estimated using an EnKF [21]. This technique is well suited for state and parameter estimation with the proposed wake model because it does not require the derivation of the tangent linear operator [21] and is computationally efficient for a discretized system of PDEs with a large number of states.

In order to apply the EnKF to the dynamic wake model, we must first reformulate the continuous problem as a discrete update equation and select a noise model to approximate modeling errors. For simplicity, we consider an explicit first-order temporal and spatial discretization of the wake model with $N_{x}$ grid points in the streamwise direction. Using this discretization, the EnKF states-composed of discretizations of the velocity deficit fields $\delta \mathbf{u}(x)$ and the wake expansion coefficient vector $\mathbf{k}$-become the following finite-dimensional column vector

$$
\boldsymbol{\psi}=\left[\boldsymbol{\delta} \mathbf{u}_{\mathbf{1}}^{T}, \ldots, \boldsymbol{\delta} \mathbf{u}_{\mathbf{N}}{ }^{T}, k_{1}, \ldots, k_{N}\right]^{T} \in \mathbb{R}^{N_{s}},
$$

where $N_{s}=\left(N_{x}+1\right) N$. Each vector $\delta \mathbf{u}_{\mathbf{n}}$ is a column vector representing the spatial discretization of $\delta u_{n}(x)$. Similarly, the column vector consisting of the measured power output of each row of turbines is denoted $\boldsymbol{\xi} \in \mathbb{R}^{N}$.

The resulting modeled wind farm system is governed by the discrete update equations

$$
\begin{aligned}
\boldsymbol{\psi}_{k+1} & =\mathbf{f}\left(\boldsymbol{\psi}_{k}, \mathbf{C}_{T k}^{\prime}\right)+\mathbf{B} \boldsymbol{\chi}_{k} \\
\boldsymbol{\xi}_{k} & =\mathbf{h}\left(\boldsymbol{\psi}_{k}, \mathbf{C}_{T k}^{\prime}\right)+\epsilon_{k}
\end{aligned}
$$

where $\boldsymbol{\psi}_{k+1}=\mathbf{f}\left(\boldsymbol{\psi}_{k}, \mathbf{C}_{T k}^{\prime}\right)$ and $\boldsymbol{\xi}_{k}=\mathbf{h}\left(\boldsymbol{\psi}_{k}, \mathbf{C}_{T k}^{\prime}\right)$ are temporal and spatial discretizations of (1)-(4) and (6)-(8), respectively. Measurement and modeling errors are represented by the white noise processes $\epsilon \in \mathbb{R}^{N}$ and $\chi \in \mathbb{R}^{2 N}$, 
respectively. All measurement noise has zero mean and equal variance $\sigma_{P}^{2}$. The process noise is subdivided into two vectors $\chi=\left[\chi_{\delta u}^{T}, \chi_{k}^{T}\right]^{T} \in \mathbb{R}^{2 N}$, where $\chi_{\delta u} \in \mathbb{R}^{N}$ has variance $\sigma_{\delta u}^{2}$ with zero mean and $\chi_{k} \in \mathbb{R}^{N}$ has variance $\sigma_{k}^{2}$ with zero mean.

In many applications, independent process noise enters all states, i.e. the identify matrix would be chosen for B. In this application, we wish to only supply one error correction term to each wake deficit equation. Therefore the error terms have a lower dimension and are distributed to each wake deficit field independently. This distribution is implemented by selecting

$$
\mathbf{B}=\left[\begin{array}{c}
\mathbf{B}_{\delta u} \\
\mathbf{I}_{N \times N}
\end{array}\right] \in \mathbb{R}^{N_{s} \times N}
$$

where $\mathbf{I}_{N \times N}$ is the identity and the matrix $\mathbf{B}_{\delta u}$ distributes $\chi_{\delta u}$ to the wake deficits $\delta \mathbf{u}$. We assume the wake deficit uncertainties are uncoupled such that

$$
\mathbf{B}_{\delta u}=\left[\begin{array}{cccc}
\mathbf{G}_{\mathbf{1}} & & & \\
& \mathbf{G}_{\mathbf{2}} & & \\
& & \ddots & \\
& & & \mathbf{G}_{\mathbf{N}}
\end{array}\right] \in \mathbb{R}^{N_{x} N \times N}
$$

where each column vector $\mathbf{G}_{\mathbf{n}} \in \mathbb{R}^{N x}$ is a spatial discretization of $G\left(x-s_{n}\right)$. The resulting noise is therefore only distributed about each turbine and uncoupled between turbine rows.

The EnKF represents the error statistics of the model using an ensemble of $N_{e}$ wake models. Each ensemble member is forced with statistically independent noise, i.e. $\chi$ and $\epsilon$ in (11)-(12). This ensemble is described by the matrix [21]

$$
\boldsymbol{\Psi}=\left[\boldsymbol{\psi}^{(1)}, \boldsymbol{\psi}^{(2)}, \ldots, \boldsymbol{\psi}^{\left(N_{e}\right)}\right] \in \mathbb{R}^{N_{s} \times N_{e}} .
$$

A corresponding ensemble of perturbed measurements is also generated. Each perturbed measurement is generated using the measurements from the true system

$$
\boldsymbol{\xi}^{(i)}=\mathbf{P}+\boldsymbol{\epsilon}^{(i)}
$$

leading to an ensemble of perturbed measurements

$$
\boldsymbol{\Xi}=\left[\boldsymbol{\xi}^{(1)}, \ldots, \boldsymbol{\xi}^{\left(N_{e}\right)}\right] \in \mathbb{R}^{N \times N_{e}},
$$

and an ensemble of measurement perturbations

$$
\mathbf{E}=\left[\boldsymbol{\epsilon}^{(1)}, \ldots, \boldsymbol{\epsilon}^{\left(N_{e}\right)}\right] \in \mathbb{R}^{N \times N_{e}} .
$$

The corresponding nonlinear outputs from the wake model ensemble are

$$
\hat{\boldsymbol{\Psi}}=\left[\mathbf{h}\left(\boldsymbol{\psi}^{1}\right), \ldots, \mathbf{h}\left(\boldsymbol{\psi}^{N_{e}}\right)\right] \in \mathbb{R}^{N \times N_{e}} .
$$

The mean of the ensemble states and outputs make up the columns of the matrices

$$
\begin{aligned}
& \overline{\mathbf{\Psi}}=\boldsymbol{\Psi} \mathbf{1}_{N_{e}} \in \mathbb{R}^{N_{s} \times N_{e}} \\
& \overline{\hat{\mathbf{\Psi}}}=\hat{\mathbf{\Psi}} \mathbf{1}_{N_{e}} \in \mathbb{R}^{N \times N_{e}}
\end{aligned}
$$

respectively, where $\mathbf{1}_{N_{e}} \in \mathbb{R}^{N_{e} \times N_{e}}$ is a full matrix whose elements equal $1 / N_{e}$. The corresponding ensemble state perturbation matrix $\Psi^{\prime}$ is

$$
\boldsymbol{\Psi}^{\prime}=\boldsymbol{\Psi}-\overline{\boldsymbol{\Psi}} \in \mathbb{R}^{N_{s} \times N_{e}},
$$

and the ensemble output perturbation matrix is $\hat{\Psi}^{\prime}$

$$
\hat{\mathbf{\Psi}}^{\prime}=\hat{\mathbf{\Psi}}-\overline{\hat{\mathbf{\Psi}}} \in \mathbb{R}^{N \times N_{e}} .
$$

The EnKF replaces the update equations (11)-(12) with two matrix update equations [21] for the EnKF wake states. This process consists of an intermediate forecast step, denoted as step $k+$, and an measurement analysis step. The ensemble matrix is forecasted using (11)

$$
\begin{aligned}
\boldsymbol{\Psi}_{k+}= & {\left[\mathbf{f}\left(\boldsymbol{\psi}_{k}^{(1)}, \mathbf{C}_{T k}^{(1)}\right)+\boldsymbol{\xi}_{k}^{(1)}, \ldots,\right.} \\
& \left.\mathbf{f}\left(\boldsymbol{\psi}_{k}^{\left(N_{e}\right)}, \mathbf{C}_{T k}^{\prime\left(N_{e}\right)}\right)+\boldsymbol{\xi}_{k}^{\left(N_{e}\right)}\right]
\end{aligned}
$$

The subsequent measurement analysis step is

$$
\begin{aligned}
\boldsymbol{\Psi}_{k+1}= & \boldsymbol{\Psi}_{k+}+\boldsymbol{\Psi}_{k+}^{\prime} \hat{\boldsymbol{\Psi}}_{k+}^{\prime T}\left(\hat{\boldsymbol{\Psi}}_{k+}^{\prime} \hat{\mathbf{\Psi}}_{k+}^{\prime T}+\right. \\
& \left.\mathbf{E}_{k+1} \mathbf{E}_{k+1}^{T}\right)^{-1}\left(\boldsymbol{\Xi}_{k+1}-\hat{\boldsymbol{\Psi}}_{k+}\right) .
\end{aligned}
$$

Following this procedure, the estimated wake model states and parameters are contained in the columns of the ensemble mean matrix $\overline{\mathbf{\Psi}}$.

\section{Receding horizon controller}

The state estimator is now used to augment the modelbased receding horizon control approach used in prior work [19], [20]. The controller is designed to modulate the turbine-local thrust coefficients of each row $\mathbf{C}_{T}^{\prime}(t)$ in order to track the power reference signal $P_{\text {ref }}(t)$. The receding horizon method is an iterative approach [12], [22], where over every time period $T_{A}$ a control trajectory is calculated for a longer time horizon $T$ by minimizing a cost functional. In this implementation we choose a time horizon of $T=10$ min, which is longer than the time it takes for the wind to travel across the wind farm, and an advancement time of $T_{A} \approx 1 \mathrm{~s}$. We use a cost functional that represents the power tracking problem by penalizing deviations from the reference power $P_{\text {ref }}(t)$

$$
\mathscr{J}=\int_{t_{0}}^{t_{0}+T}\left(\sum_{n=1}^{N} \hat{P}_{n}(t)-P_{\text {ref }}(t)\right)^{2} d t,
$$

where $t_{0}$ is the current time.

Using this cost function, we solve the following minimization problem

$$
\begin{array}{ll}
\underset{\boldsymbol{\varphi}(t), \mathbf{q}(x, t)}{\operatorname{minimize}} & \mathscr{J}(\mathbf{q}(x, t)) \\
\text { subject to } & \mathbf{W}(\mathbf{q}(x, t))=\mathbf{0} \\
& \frac{d C_{T n}^{\prime}}{d t}=\frac{1}{\tau}\left[\varphi_{n}(t)-C_{T n}^{\prime}(t)\right] \\
& 0 \leq \varphi_{n}(t) \leq 2 .
\end{array}
$$

where $\mathbf{q}(x, t)=\left[\boldsymbol{\delta} \mathbf{u}(x, t), \hat{\mathbf{u}}(t), \mathbf{C}_{T}^{\prime}(t)\right], \boldsymbol{\varphi}(t)$ are auxiliary control variables, and $\mathbf{W}(\mathbf{q}(x, t))=\mathbf{0}$ represents the wake 
model discussed above. The auxiliary control variables $\varphi(t)$, whose rate of change is unconstrained, is used to prevent high-frequency oscillations of the turbine-local thrust coefficient. By specifying $\mathbf{C}_{T}^{\prime}(t)$ through a first-order relaxation of $\boldsymbol{\varphi}(t)$ with a time constant $\tau$, the rate of change of $\mathbf{C}_{T}^{\prime}(t)$ is implicitly limited. Furthermore, we select bounds on the control variables of $\varphi \in[0,2]$ to prevent the turbine-local thrust coefficient from becoming negative or exceeding the Betz limit of $C_{T}^{\prime}=2$ [23].

Instead of directly minimizing the constrained cost functional, the tracking problem is solved by minimizing the modified unconstrained reduced cost function $\tilde{\mathscr{J}}(\varphi)$. Minimization is performed using the limited-memory boundconstrained quasi-Newton method L-BFGS-B [24]. Gradients are evaluated using backward simulations of the analytically-derived adjoint equations to the constraints (28) and (29). The adjoint equations are discussed in more detail in [19].

\section{NUMERICAL RESULTS}

In this section we test the state estimation and the closedloop controlled wind farm using a high fidelity simulation as the wind farm plant. In particular, we use LES of a wind farm, in which the filtered Navier-Stokes equations are solved using JHU's LESGO code with wind turbines represented using actuator disks [7], [5], [25]. The simulations use periodic spanwise boundary conditions with psuedo-spectral discretization for the streamwise and spanwise directions and second-order finite differencing for the vertical direction. Time integration is performed using the explicit second-order Adams-Bashforth method.

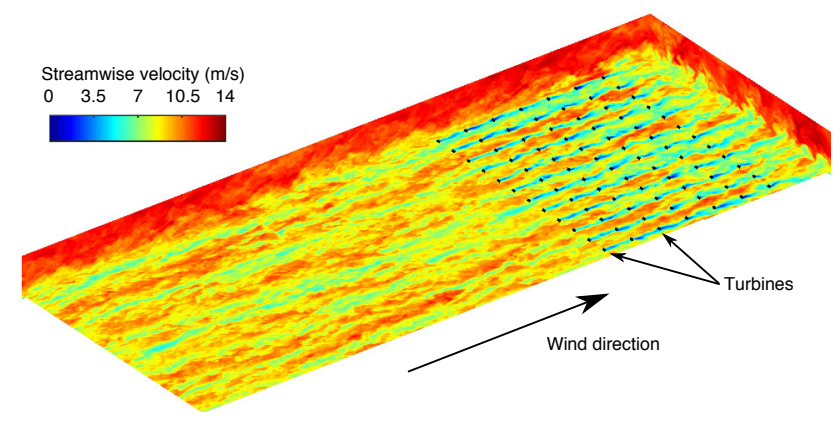

Fig. 4. Instantaneous color contour plot of the velocity field in a sample wind farm LES. The prevailing (streamwise) wind direction is from left to right. The precursor domain at the beginning of the figure shows the high fidelity representation of atmospheric boundary layer turbulence. Wind turbines are shown in black at the end of the domain, and low speed regions (wakes) are apparent behind each turbine. (Adapted from [20].)

In all simulations, an 84-turbine wind farm with 7 rows of 12 aligned turbines is considered. The wind farm simulation domain is $9 \mathrm{~km} \times 6 \mathrm{~km} \times 1 \mathrm{~km}$ in length, width and height, respectively, with $384 \times 256 \times 192$ grid points in each direction. We employ the concurrent-precursor method [25], which uses a separate fully-developed atmospheric boundary layer simulation with identical geometry, but without turbines, to generate the inlet conditions. An instantaneous color contour plot of the combined precursor and simulation domains for one of these simulations is shown in Figure 4.

\section{A. State estimation}

The state and parameter estimation of the wake velocity deficits $\delta u_{n}(x, t)$, wake expansion coefficients $k_{n}(t)$, and freestream velocity $U_{\infty}(t)$, discussed in Section III-B, is tested using measurements from three LES with independent initial conditions. For each test, the wake expansion coefficients are all initially set to the same value of $k_{n}=0.05$. The standard deviation of the state and output perturbations$\sigma_{k}=0.0001, \sigma_{\delta u}=0.05 \mathrm{~m} / \mathrm{s}$, and $\sigma_{P}=0.29 \mathrm{MW}$-are tuned to provide good estimation performance. An ensemble of 250 members is generated by forcing with random noise terms. Each noise term is a normally-distributed number proportional to the standard deviation times the square-root of the step size [21]. The initial error distributions are formed by integrating each member forward in time [21] for one advective time scale of the entire farm.

To examine the error in power estimation, we use the average relative estimation error measure $\frac{1}{T} \int_{0}^{T} \mid \hat{P}_{n}(t)-$ $P_{n}(t) \mid / P_{n}(t) d t$ for each row, where $P_{n}(t)$ is the measured power in LES and $\hat{P}_{n}(t)$ is the estimated power. Table I shows the average relative estimation error for all initial conditions. Instantaneous plots of the measured power from LES, the estimated power, and the relative error by row are shown in Figure 5. Taken together, these results demonstrate that the instantaneous relative error does not exceed $4 \%$ and the average relative estimation is always less than $1.5 \%$ for all rows.

TABLE I

AVERAGE RELATIVE ESTIMATION ERROR OF POWER $\frac{1}{T} \int_{0}^{T}\left|\hat{P}_{n}-P_{n}\right| / P_{n} d t(\%)$ B R ROW $n$.

\begin{tabular}{|c||c||c||c||c||c||c||c|}
\hline $\mathrm{n}$ & 1 & 2 & 3 & 4 & 5 & 6 & 7 \\
\hline Test 1 & 0.26 & 1.23 & 0.39 & 0.46 & 0.37 & 0.33 & 0.41 \\
\hline Test 2 & 0.22 & 0.80 & 0.41 & 0.42 & 0.39 & 0.35 & 0.35 \\
\hline Test 3 & 0.23 & 0.68 & 0.41 & 0.47 & 0.38 & 0.37 & 0.37 \\
\hline
\end{tabular}

The estimated values of the wake expansion coefficients are shown in Figure 6. For each initial condition, the wake expansion coefficients are compared to a best-fit estimate of these coefficients from the average power of each row over the entire validation window. This best-fit is performed after the simulation and assumes a constant wake expansion rate for each turbine row for the entire window. For each initial condition, these best-fit coefficients demonstrate that the wake expansion rate is lower $k_{1} \approx 0.03$ for the first row than subsequent rows $k_{n} \approx 0.05$. For the last two initial conditions, we see that the estimated wake expansion coefficient approaches the distribution of wake expansion coefficients as expected from the best fit. The last case, however, does not approach the expected distribution, and more exploration is needed to study this case.

The wake model's velocity field $u(x, t)$ defined in (6) is compared to the LES velocity field $\tilde{u}\left(x, y, z_{h}, t\right)$ at the 


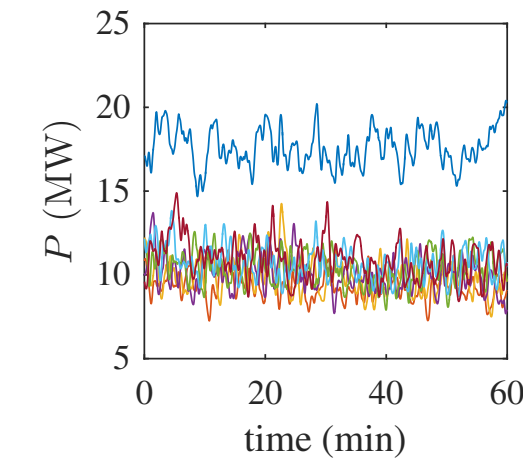

Row 1
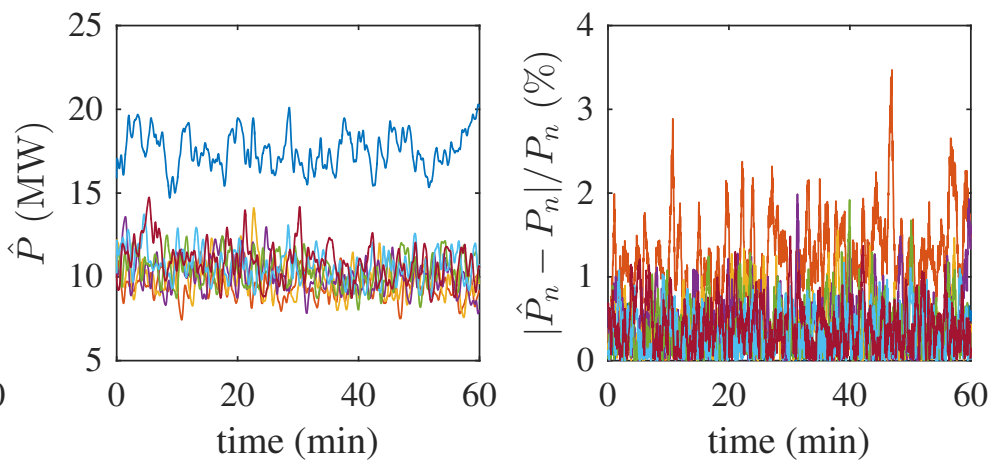

Row 5

Row 6

Row 7

Fig. 5. Power generation of wind farm rows in LES (left) and the EnKF (center), as well as the instantaneous relative error of the wake model estimation for each row (right).

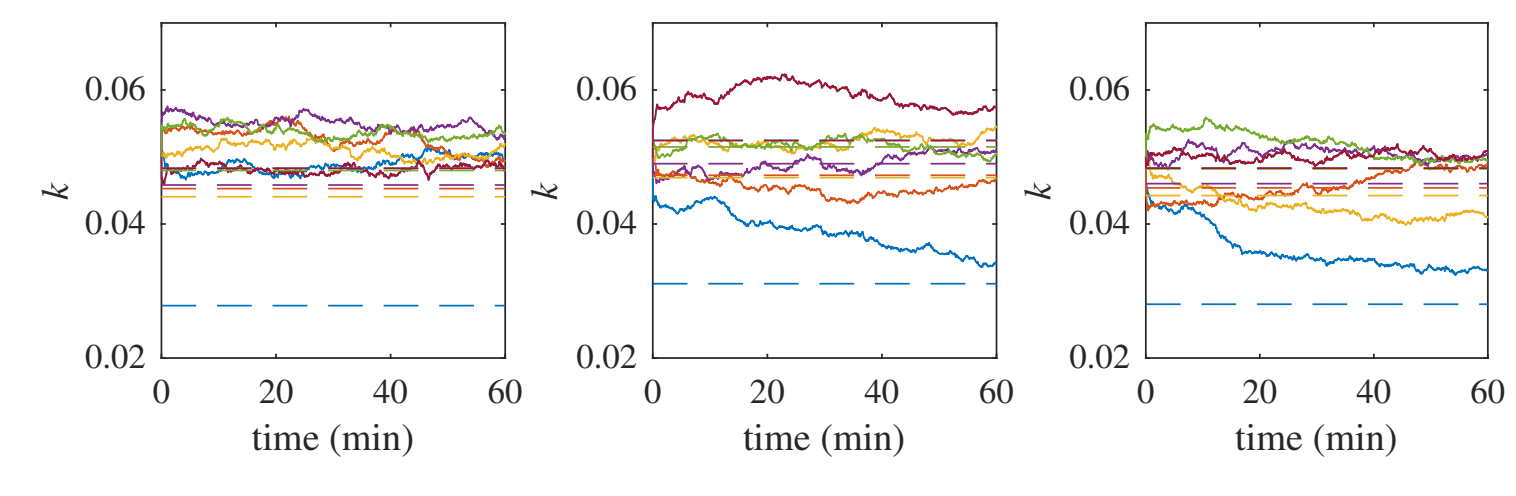

Row $1-$ Row $2-$ Row $3-$ Row $4-$ Row $6-$ Row 7

Fig. 6. Comparison of wake expansion coefficients by row as calculated using EnKF ( - ) and ex post facto best fit of mean power generation using the wake model $(---)$. Each panel shows a different initial condition. Two of the three panels capture the substantial difference between the wake expansion rates of the first row and subsequent rows.

height of the turbine rotor $z_{h}$. In order to compare the modeled streamwise velocity along each turbine row, the row-averaged LES velocity field is computed using

$$
\langle\tilde{u}\rangle(x, t)=\frac{1}{D M} \int_{0}^{L_{y}}\left[\sum_{\tilde{m}=1}^{M} H\left(\frac{D}{2}-\left|y-y_{m}\right|\right)\right]
$$

where $H(y)$ is the Heaviside function and $y_{m}$ is the location of the $m$-th column of turbines. Figure 7 compares a modeled velocity field using the EnKF $u(x, t)$ to a row-averaged streamwise velocity profile from LES $\langle\tilde{u}\rangle(x, t)$. This velocity field comparison demonstrates good correspondence between the measured and estimated velocity field near the location of each turbine and captures the changing wake expansion rates and advection of the velocity deficits. As expected, the errors increase downstream of each turbine where measurements are not available.

\section{B. Controlled wind farm}

The controlled wind farm is tested in twelve total simulations. We refer to the five-minute pre-control average

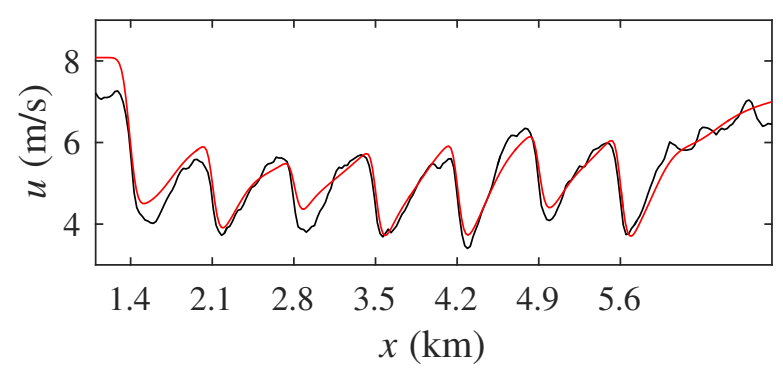

Fig. 7. Instantaneous row-averaged velocity profile from LES ( -$)$, as defined in (31) and the EnKF wake model estimate ( - ), as defined in (6). Each tick mark denotes a turbine row location.

power as $P_{\text {base. }}$ The regulation signal can then be defined as $P_{\text {ref }}(t)=[1-\alpha+\beta r(t)] P_{\text {base }}$. In order to track the signal, the wind farm power set point must be reduced by some fraction $\alpha$, which is referred to as the derate. The signal $r(t) \in[-1,1]$ is the reference signal sent by the grid operator that is scaled as a fraction $\beta$ of the pre-control power $P_{\text {base }}$.

In these tests we use a reference signal with $\beta=0.08$ and 

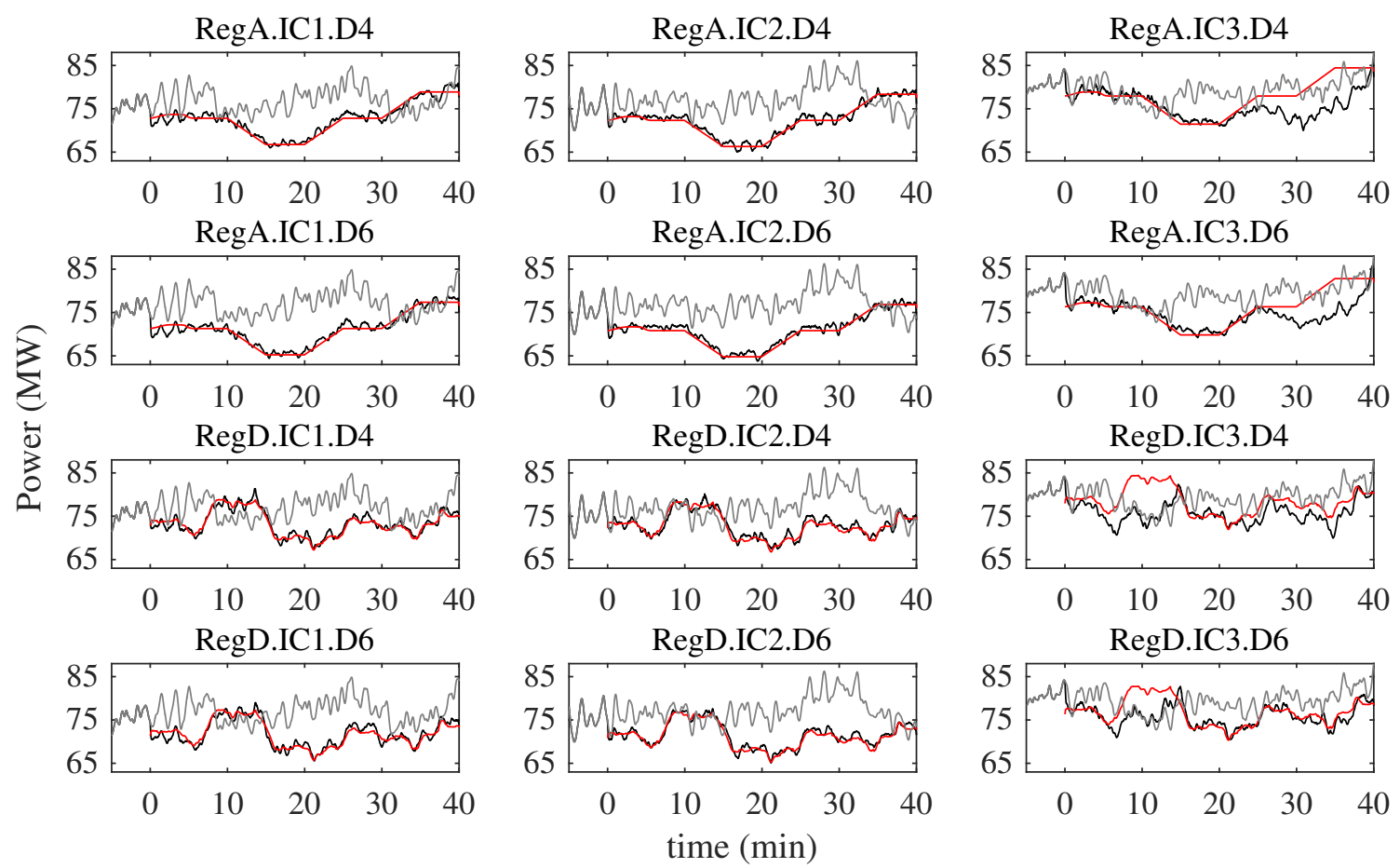

Fig. 8. Controlled wind farm power output ( - ) compared to reference signal ( - ) and uncontrolled wind farm power output $(-)$. All twelve simulations are shown and denoted by the signal type (RegA or RegD), the initial conditions (IC1-IC3), and derate (D4 for 4\% and D6 for 6\%).

derates $\alpha=0.04$ and 0.06 . The signal $r(t)$ is taken from PJM, an independent system operator in the US. Test signals from PJM's published traditional RegA and fast-acting RegD markets are used [26], [27]. Furthermore, each derate and signal type combination is tested using all three ending states of the EnKF test cases.

The performance of the controlled wind farm is shown for all twelve simulations in Figure 8. Each panel shows the reference signal as described above, the controlled power output, and the uncontrolled power output if the pre-control thrust coefficient $C_{T}^{\prime}=1.33$ were continued from $t=0$ using the same wind farm state. The performance of the controlled wind farm under the first two initial conditions demonstrate good tracking performance for the the slowlyvarying RegA signals as well as the fast-acting RegD signal. The rate-limiting of the control actuation filter results in noticeable fluctuations around the requested power reference signal and some overshoot at the beginning of the control period. However, the controlled farm power has noticeably smaller fluctuations than the turbulent fluctuations of the uncontrolled power. By reducing turbulent fluctuations, the wind farm behaves more like a conventional generator by producing more consistent power output to the grid.

These simulations also demonstrate the importance of including a dynamic wake model into the control design. During some periods of the simulation-such as around the 10-minute mark of the RegD.IC1.D4 signal and the last 5 minutes of the RegA.IC2.D4 signal-the controlled wind farm was able to produce more power than the uncontrolled farm. Similar trends were seen in prior work [19], [20], where the controller is able to reduce the energy extraction of upstream turbines during periods with more available energy in the flow field, thereby providing increased power production potential for downstream rows. This mechanism would allow for increased production for a short time duration by deferring upstream wind turbine energy extraction. Characterizing this phenomena is a direction for continuing study.

The relatively poor tracking performance of the third initial condition requires further investigation. The underproduction during the last twenty minutes of the RegA cases with the third initial condition is hard to explain. The uncontrolled farm produces more energy than requested by the reference signal prior to the period. Furthermore, the uncontrolled wind farm produces more power than the controlled farm in these cases; clearly there is enough kinetic energy in the flow field to meet the power reference signal. Determining the cause of this behavior is the focus of ongoing work.

On the other hand, the under-production around the 10 minute mark of the RegD.IC3.D4 and RegD.IC3.D6 signals in Figure 8 might be explained by insufficient energy availability. The uncontrolled farm produces less power than requested by the reference signal, and the wind farm may be unavailable to accommodate the significant increased production requested by the grid operator. In other words, 
there simply may not be enough available kinetic energy in the flow field to provide the required level of power during this time period.

\section{CONCLUSIONS}

The performance of model-based receding horizon control for wind farm power tracking applications was considered. This approach used a new dynamic wake model that accounts for important flow phenomena such as wake advection and interactions. An ensemble Kalman filter was used to provide state and parameter estimation. The state estimation was validated against measurements from LES. The addition of the EnKF provides a more practical approach for estimating wake model parameters and correcting wake model states because parameters do not have to be fit prior to initiation of the control. The addition of the auxiliary control eliminates the need for regularizations of the cost functional. Results demonstrate the effectiveness of the controlled wind farm in tracking sample reference signals from PJM. While the controlled farm failed to track the signal for one inflow condition, the other inflow conditions showed good tracking performance.

\section{REFERENCES}

[1] J. Aho, A. Buckspan, J. Laks, P. Fleming, Y. Jeong, F. Dunne, M. Churchfield, L. Pao, and K. Johnson, "A tutorial of wind turbine control for supporting grid frequency through active power control," in American Control Conference, June 2012, pp. 3120-3131.

[2] F. Díaz-González, M. Hau, A. Sumper, and O. Gomis-Bellmunt, "Participation of wind power plants in system frequency control: Review of grid code requirements and control methods," Renewable and Sustainable Energy Reviews, vol. 34, pp. 551-564, 2014.

[3] J. Annoni, K. Howard, P. Seiler, and M. Guala, "An experimental investigation on the effect of individual turbine control on wind farm dynamics," Wind Energy, 2015.

[4] P. A. Fleming, P. M. Gebraad, S. Lee, J.-W. van Wingerden, K. Johnson, M. Churchfield, J. Michalakes, P. Spalart, and P. Moriarty, "Evaluating techniques for redirecting turbine wakes using SOWFA," Renewable Energy, vol. 70, pp. 211-218, 2014.

[5] C. VerHulst and C. Meneveau, "Altering kinetic energy entrainment in large eddy simulations of large wind farms using unconventional wind turbine actuator forcing," Energies, vol. 8, no. 1, pp. 370-386, 2015.

[6] R. J. Stevens and C. Meneveau, "Flow structure and turbulence in wind farms," Annual Review of Fluid Mechanics, vol. 49, no. 1, 2017.

[7] M. Calaf, C. Meneveau, and J. Meyers, "Large eddy simulation study of fully developed wind-turbine array boundary layers," Physics of Fluids, vol. 22, no. 1, 2010.

[8] J. Meyers and C. Meneveau, "Large eddy simulations of large windturbine arrays in the atmospheric boundary layer," in Proceedings of 50th AIAA Aerospace Sciences Meeting, 2010.
[9] I. Katić, J. Højstrup, and N. Jensen, "A simple model for cluster efficiency," in European Wind Energy Association Conference and Exhibition, 1986, pp. 407-410.

[10] S. Frandsen, R. Barthelmie, S. Pryor, O. Rathmann, S. Larsen, J. Højstrup, and M. Thøgersen, "Analytical modelling of wind speed deficit in large offshore wind farms," Wind Energy, vol. 9, no. 1-2, pp. 39-53, 2006.

[11] R. J. A. M. Stevens, D. F. Gayme, and C. Meneveau, "Coupled wake boundary layer model of wind-farms," Journal of Renewable and Sustainable Energy, vol. 7, no. 2, 2015.

[12] J. P. Goit and J. Meyers, "Optimal control of energy extraction in wind-farm boundary layers," Journal of Fluid Mechanics, vol. 768, pp. 5-50, 2015.

[13] J. Ainslie, "Calculating the flowfield in the wake of wind turbines," Journal of Wind Engineering and Industrial Aerodynamics, vol. 27, no. $1-3$, pp. $213-224,1988$.

[14] M. Bastankhah and F. Porté-Agel, "A new analytical model for windturbine wakes," Renewable Energy, vol. 70, pp. 116-123, 2014.

[15] P. Fleming, J. Aho, P. Gebraad, L. Pao, and Y. Zhang, "Computational fluid dynamics simulation study of active power control in wind plants," in American Control Conference, July 2016, pp. 1413-1420.

[16] G. C. Larsen, H. A. Madsen, K. Thomsen, and T. J. Larsen, "Wake meandering: a pragmatic approach," Wind Energy, vol. 11, no. 4, pp. 377-395, 2008.

[17] J. Annoni, P. Gebraad, and P. Seiler, "Wind farm flow modeling using an input-output reduced-order model," in American Control Conference, July 2016, pp. 506-512.

[18] P. M. Gebraad and J. Van Wingerden, "A control-oriented dynamic model for wakes in wind plants," in Journal of Physics: Conference Series, vol. 524, no. 1, 2014.

[19] C. R. Shapiro, P. Bauweraerts, J. Meyers, C. Meneveau, and D. F. Gayme, "Model-based receding horizon control of wind farms for secondary frequency regulation," Wind Energy, 2016. Submitted.

[20] C. R. Shapiro, J. Meyers, C. Meneveau, and D. F. Gayme, "Wind farms providing secondary frequency regulation: Evaluating the performance of model-based receding horizon control," Journal of Physics: Conference Series, vol. $\mathrm{xx}$, no. $\mathrm{xx}, \mathrm{pp} . \mathrm{xxx}-\mathrm{xxx}, 2016$.

[21] G. Evensen, "The ensemble kalman filter: theoretical formulation and practical implementation," Ocean Dynamics, vol. 53, no. 4, pp. 343367, 2003.

[22] T. R. Bewley, P. Moin, and R. Temam, "DNS-based predictive control of turbulence: an optimal benchmark for feedback algorithms," Journal of Fluid Mechanics, vol. 447, pp. 179-225, 2001.

[23] J. Meyers, W. Munters, and J. Goit, "A framework for optimization of turbulent wind-farm boundary layers and application to optimal control of wind-farm energy extraction," in American Control Conference, July 2016, pp. 519-524.

[24] C. Zhu, R. H. Byrd, P. Lu, and J. Nocedal, "Algorithm 778: L-BFGSB: Fortran subroutines for large-scale bound-constrained optimization," ACM Transactions on Mathematical Software, vol. 23, no. 4, pp. 550-560, 1997.

[25] R. J. A. M. Stevens, J. Graham, and C. Meneveau, "A concurrent precursor inflow method for large eddy simulations and applications to finite length wind farms," Renewable Energy, vol. 68, pp. 46-50, 2014.

[26] PJM, PJM Manual11: Energy \& Ancillary Services Market Operations, 2012.

[27] — PJM Manual 12: Balancing Operations, 2015. 